\title{
Gain of Rod to Horizontal Cell Synaptic Transfer: Relation to Glutamate Release and a Dihydropyridine-Sensitive Calcium Current
}

\author{
Paul Witkovsky, ${ }^{1,2}$ Yvonne Schmitz, ${ }^{1}$ Abram Akopian, ${ }^{1}$ David Krizaj, ${ }^{1,2}$ and Daniel Tranchina ${ }^{3}$ \\ Departments of ${ }^{1}$ Ophthalmology and ${ }^{2}$ Physiology and Neuroscience, New York University Medical Center, New York, \\ New York 10016, and ${ }^{3}$ Departments of Biology and Mathematics and the Center for Neural Science, New York University, \\ New York, New York 10003
}

\begin{abstract}
We related rod to horizontal cell synaptic transfer to glutamate release by rods. Simultaneous intracellular records were obtained from dark-adapted rod-horizontal cell pairs. Steadystate synaptic gain (defined as the ratio of horizontal cell voltage to rod voltage evoked by the same light stimulus) was $3.35 \pm 0.60$ for dim flashes and $1.50 \pm 0.03$ for bright flashes. Under conditions of maintained illumination, there was a measurable increment of horizontal cell hyperpolarization for each light-induced increment of rod hyperpolarization over the full range of rod voltages.

In separate experiments we studied glutamate release from an intact, light-responsive photoreceptor layer, from which in-
\end{abstract}

ner retinal layers were removed. Steady light reduced glutamate release as a monotonic function of intensity; spectral sensitivity measures indicated that we monitored glutamate release from rods. The dependence of glutamate release on rod voltage was well fit by the activation function for a high-voltage-activated, dihydropyridine-sensitive L-type calcium current, suggesting a linear dependence of glutamate release on $[\mathrm{Ca}]_{i}$ in the synaptic terminal. A simple model incorporating this assumption accounts for the steady-state gain of the rod to horizontal cell synapse.

Key words: Xenopus; photoreceptor; rod; synaptic gain; horizontal cell; glutamate release; calcium
In vertebrate retinas, the photoreceptors (rods and cones) and the second-order retinal neurons (horizontal and bipolar cells) all are nonspiking neurons with light-evoked responses that are slow potentials of complex waveform, graded in amplitude with stimulus intensity. Glutamate, the transmitter used by both rods and cones (Copenhagen and Jahr, 1989; Marc et al., 1990) is released at a steady rate in darkness (Schmitz and Witkovsky, 1996). Light, by hyperpolarizing the photoreceptor membrane, decreases glutamate release (for review, see $\mathrm{Wu}, 1994$ ).

At synapses between spiking cells, types $\mathrm{N}$ and $\mathrm{P}$ calcium channels are most often implicated in the gating of neurotransmitter release (for review, see Olivera et al., 1994; Regehr and Mintz, 1994; Katz et al. 1995). In contrast, at a tonic retinal synapse for which a depolarizing bipolar cell is the presynaptic element, transmitter release is controlled by a dihydropyridinesensitive L-type calcium current (Heidelberger and Matthews, 1992; Tachibana et al., 1993). Rods and cones also possess an L-type Ca current (Bader et al., 1982; Corey et al., 1984; Barnes and Hille, 1989; Lasater and Witkovsky, 1991; Wilkinson and Barnes, 1996), and there is evidence that it underlies exocytosis

Received March 25, 1997; revised June 2, 1997; accepted July 18, 1997.

This work was supported by the Helen Hoffritz foundation, an unrestricted award from Research to Prevent Blindness, Inc., to the Department of Ophthalmology, New York University Medical Center, by Grant EY 03570 and a Senior Scientific Investigator Award from Research to Prevent Blindness, Inc., to P.W., and by a Deutsche Forschungsgemeinschaft fellowship to Y.S.

Correspondence should be addressed to Dr. Paul Witkovsky, Department of Ophthalmology, New York University Medical Center, 550 First Avenue, New York, NY 10016.

Dr. Krizaj's present address: Department of Ophthalmology, University of California, San Francisco, CA 94143.

Copyright (C) 1997 Society for Neuroscience $0270-6474 / 97 / 177297-10 \$ 05.00 / 0$
(Rieke and Schwartz, 1996) and glutamate release (Schmitz and Witkovsky, 1997). In contrast to the depolarizing bipolar cell, however, light hyperpolarizes the photoreceptor, bringing its membrane into a voltage range $(<-45 \mathrm{mV})$ in which the L-type $\mathrm{Ca}$ current, as characterized by whole-cell patch-clamp recordings (Corey et al., 1984; Wilkinson and Barnes, 1996), becomes too small to measure. A main concern of the present study is whether the L-type Ca current can control transmitter release over the full range of rod light-induced hyperpolarizations, or whether an additional calcium current may be required, for example, the one in cone photoreceptors that depends on cGMP (Rieke and Schwartz, 1994).

We explored this question in two ways. In one, we used a reduced retina preparation consisting primarily of an intact photoreceptor layer and the subjacent retinal pigment epithelium (Schmitz and Witkovsky, 1996, 1997) to measure glutamate release and to study its dependence on light and its relation to the membrane potential of the rod photoreceptor. In the second, we recorded simultaneously from rods and horizontal cells, under conditions in which cone input to the horizontal cell (HC) was excluded. These two data sets permitted us to relate synaptic gain to transmitter release by the rod. Our electrophysiological and glutamate release data, in combination with the activation function for an L-type Ca calcium current (Corey et al., 1984), suggest that this $\mathrm{Ca}$ current underlies rod to $\mathrm{HC}$ synaptic transfer over the full voltage range of rod responses evoked by steady illumination.

\section{MATERIALS AND METHODS}

Animals. We used adult, male Xenopus laevis, obtained from NASCO (Ft. Atkinson, WI) and maintained in an aerated aquarium on a $12 \mathrm{~h}$ 
light/dark cycle, lights on at 6 A.M. Light-adapted frogs were anesthetized with $0.2-0.3 \mathrm{mg}$ of tricaine methanesulfonate (Sigma, St. Louis, $\mathrm{MO}$ ) in $0.3 \mathrm{cc}$ of Ringer's solution, given subcutaneously. In room light, the eye was excised, the anterior portion was cut away, and the posterior pole was pinned flat to a wax chamber.

Intracellular recording. The procedures for intracellular recording are described in detail elsewhere (Krizaj et al., 1994). Briefly, for the intracellular experiments we used a bicarbonate Ringer's solution, $\mathrm{pH} 7.4$, which was superfused over the eyecup at $1.5 \mathrm{ml} / \mathrm{min}$. Intracellular records were obtained with sharp microelectrodes using standard procedures. The data were stored on digital tape and processed off-line using Modular Instruments (Southeastern, PA) hardware and Spike software. The irradiances of light stimuli were measured with a photodiode in the plane of the retina, referenced to a calibrated thermopile, and are given as $\log$ incident quanta $\mathrm{cm}^{-2} / \mathrm{sec}$.

Adaptational state. We obtained simultaneous records from rods and horizontal cells, either under mesopic conditions, i.e., when the lightevoked response of the $\mathrm{HC}$ revealed both rod and cone input, or under scotopic conditions, meaning that the $\mathrm{HC}$ was driven by rods alone. The state of visual adaptation was achieved by the time in the light/dark cycle when the experiment began and the time the eyecup preparation was left in darkness thereafter. For mesopic conditions the preparation was placed in darkness about noon. HC responses were monitored (typically 2-3 hr) until the appropriate balance of rod and cone inputs was observed. To achieve scotopic recording conditions we began experiments in the afternoon and allowed the preparation at least $4 \mathrm{hr}$ of dark adaptation.

Estimation of synaptic gain. For the data of Figures 3-5, rod and HC waveforms were transferred from digital tape to Spike software with a sampling rate of $0.18 \mathrm{kHz}$. A movable cursor read the trace voltage at any sampling point. Typical maximum voltages were (compare Fig. 1) rod plateau, $14 \mathrm{mV}$; HC plateau (mesopic), $10 \mathrm{mV}$; and HC peak (scotopic; compare Fig. 2), $20 \mathrm{mV}$. Data from different cell pairs were normalized to these values. For Figures 4 and 5, response amplitudes were measured each $150 \mathrm{msec}$ in regions of relatively rapid voltage change and each 500 msec during maintained plateaus.

Glutamate release. Glutamate release by photoreceptors was measured using a "reduced retina" preparation (Cahill and Besharse, 1992), as described by Schmitz and Witkovsky (1996). The inner retinal layers were separated from the photoreceptor layer by exposing the eyecup successively, for $1.5 \mathrm{~min}$ each, to $0.5 \%$ Triton X-100 and distilled water. Within $1 \mathrm{hr}$ of incubation in medium (bicarbonate Ringer's solution enriched with 14 amino acids; Cahill and Besharse, 1991), the retina splits in the middle of the inner nuclear layer, allowing the inner retina to be removed with forceps. The posterior pole of the eye, consisting of photoreceptor layer and adjacent pigment epithelial and choroidal layers, was superfused with the above medium at $1 \mathrm{ml} / \mathrm{hr}$; the superfusate was collected at $10 \mathrm{~min}$ intervals, and its glutamate content was measured via an enzyme assay, which couples glutamate dehydrogenase and FMN reductase (both from Boehringer Mannheim, Indianapolis, IN) to bacterial luciferase (Sigma) (Fosse et al., 1986). In an earlier study (Schmitz and Witkovsky, 1996) we found that glutamate release in darkness was about twofold greater than in bright light. These measures were obtained in the absence of a glutamate uptake blocker. In the present study we examined glutamate release in the presence of a glutamate uptake blocker, $1 \mathrm{~mm}$ dihydrokainate (for review, see Danbolt, 1994). This substance was without effect on the rate of glutamate release in bright light, but it increased glutamate efflux in darkness, such that dark release exceeded that in bright light by a factor of $2.84 \pm 0.20 ; n=12$. All experiments on glutamate release were done in the presence of $1 \mathrm{~mm}$ dihydrokainate.

\section{RESULTS}

\section{Simultaneous recording from rod-HC pairs}

In amphibian retinas, second-order neurons receive direct synaptic input from both rods and cones (Hanani and Vallerga, 1980; Hare and Owen, 1995). In an earlier study we showed (Witkovsky et al., 1989) that rod and cone inputs to the HC are not independent. Here we explore whether a cone input to the horizontal cell can influence the apparent gain of the rod to HC synapse.

Figure 1 shows the light-evoked responses of a rod-HC pair in a mesopic retina. As stimulus intensity is increased (in $0.4 \mathrm{log}$

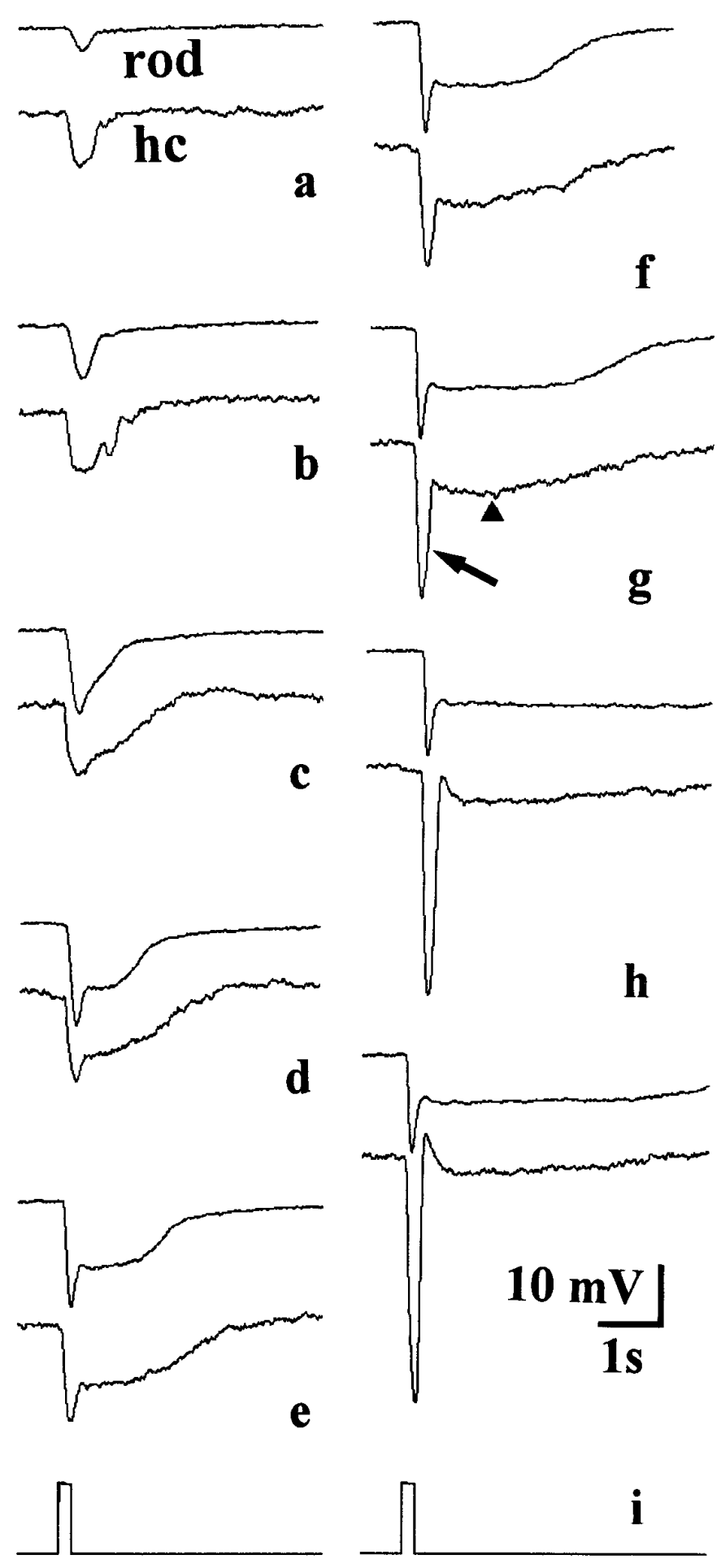

Figure 1. Intracellular recording from a rod-HC pair in mesopic state. For each pair of traces in Figures 1 and 2, the top recording is from a rod, and the bottom recording is from an HC. The lowest trace in each column indicates the timing of a $200 \mathrm{msec}$ flash. Stimulus intensity of a $567 \mathrm{~nm}$ flash increases from $a$ to $h$ in $0.4 \log$ unit steps. For $a, \log$ quanta incident $\mathrm{cm}^{-2} / \mathrm{sec}=9.9$. Note the increase in cone-dependent transient ( $g$, arrow) and the decrease in rod-dependent plateau ( $g$, triangle $)$ of $\mathrm{HC}$ response as stimulus intensity increases. 


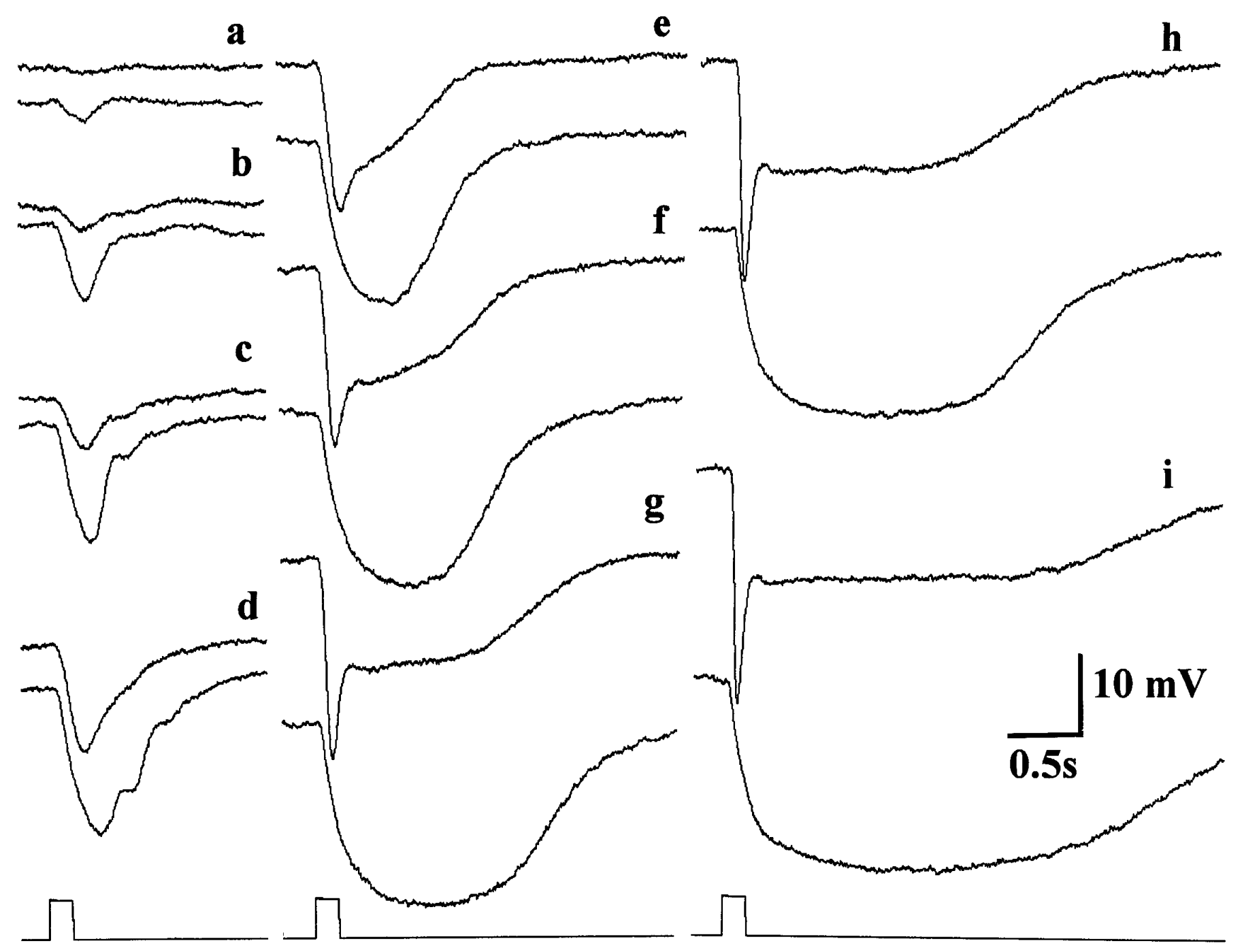

Figure 2. Intracellular recording from a rod-HC pair in scotopic state. Rod-HC pairs are as in Figure 1 . Stimulus intensity of a $567 \mathrm{~nm}$ flash (200 msec) increases from $a$ to $i$ in $0.4 \log$ unit steps. Log quantal flux in $a$ is 9.1. Note that the HC waveform lacks an initial transient.

increments from $a$ to $i$ ), the cone-dependent transients (one illustrated by an arrow, record $g$ ) of the $\mathrm{HC}$ responses increase in amplitude, whereas the subsequent rod-dependent plateaus ( $g$, triangle) decrease. Comparable intensity response data from rod-HC pairs were obtained under scotopic conditions (Fig. 2). To ensure that only rods provided input to the $\mathrm{HC}$, we used a null test in which 567 and $660 \mathrm{~nm}$ lights were adjusted in intensity for equal rod stimulation and then presented as $1 \mathrm{~Hz}$ sinusoids in counterphase. The rod responds to this complex stimulus with a non-oscillating DC shift, as does any retinal neuron receiving synaptic input from rods alone (data not shown; for examples, cf. Krizaj and Witkovsky, 1993).

Figures 1 and 2 illustrate that although rod waveforms are quite similar in mesopic or scotopic retinas, the $\mathrm{HC}$ waveform changes markedly. In scotopic retinas, $\mathrm{HC}$ light-evoked waveforms lack an initial transient, indicating a loss of cone input, and HC kinetics are slowed. From the data of Figures 1 and 2 we can obtain an estimate of synaptic gain from the ratios of rod and $\mathrm{HC}$ responses elicited by identical stimuli. All the estimates of gain in this study ignore the initial rod transient and the temporally corresponding portion of the $\mathrm{HC}$ response. This is because the glutamate release measures were obtained under conditions of steady illumination (see below). The model we developed to relate gain, glutamate release, and its underlying calcium current thus was based on steady-state conditions.

In mesopic retinas we measured the response maxima elicited by weak flashes (Fig. $1 a-c$ ); for brighter flashes (Fig. $1 d-i$ ), which elicited an initial transient in both rod and $\mathrm{HC}$ responses, we measured the maximum value of the subsequent plateaus. For scotopic retinas we took the ratio of $\mathrm{HC}$ response maximum to rod plateau. These data are shown in Figure 3. The effective operating range of mesopic rods along the intensity axis of Figure 3 is about $0.8 \log$ unit less sensitive than for scotopic rods, but this difference has been eliminated by a lateral shift of the data. In mesopic retinas, (Fig. 3, open symbols; $n=8$ ) rod-HC synaptic gain falls from $3.94 \pm 0.86$ (mean \pm SEM) for the responses of the rod-HC pair to the weakest effective test light to $0.61 \pm 0.08$ for a saturating flash. The closed circles of Figure 3 show the corresponding mean values for scotopic retinas $(n=14)$. The data indicate that for the weakest test flash, gain is not significantly different between mesopic and scotopic states. However, for all brighter stimuli, the gain is higher under scotopic condi- 


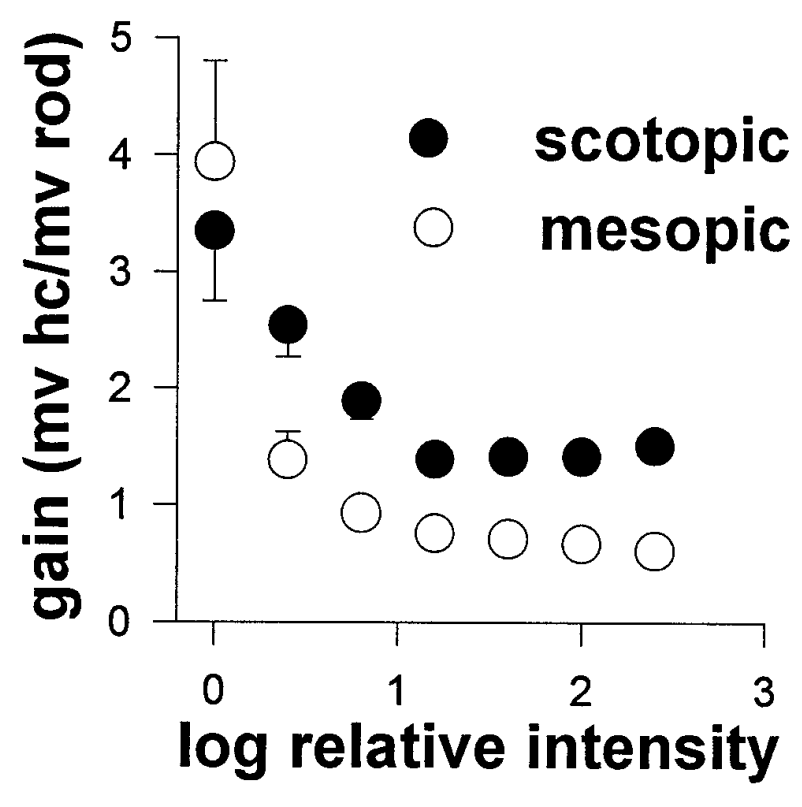

Figure 3. Gain of rod to $\mathrm{HC}$ synapse in mesopic and scotopic retinas. Gain was estimated from the ratio of $\mathrm{HC}$ to rod voltages evoked by light stimuli of different intensity, as illustrated in Figures 1 and 2. Data points show the mean \pm SEM. Log 0 on the scale of abscissa corresponds to the responses illustrated in Figures $1 a$ and $2 a$. See Results for description of voltage measurements.

tions. Moreover, in the mesopic state, gain continues to decline as stimulus intensity increases, a finding consistent with earlier data showing that increasing cone inputs to the HC tend to suppress a temporally coincident rod input (Witkovsky et al., 1989; Krizaj et al., 1994). In scotopic retinas, in contrast, the gain of rod to $\mathrm{HC}$ synaptic transfer reaches a stable minimum value of about 1.5.

Another way of representing synaptic gain is to plot the ratio of $\mathrm{HC}$ to rod voltage at multiple time points taken from the simultaneously recorded responses of these two cell types elicited by the same stimulus. Figure 4 illustrates data taken from five completely dark-adapted rod-HC pairs. Figure 4, inset, illustrates the method; for each cell pair, data from weak, intermediate, and bright flashes were superimposed. It is noteworthy that the data points from different response pairs cluster around a single line with a slope that is a measure of the gain of the synapse. The function that describes this line is derived in a mathematical model (see below and Fig. 7). The data of Figure 4 show that the $\mathrm{HC}$ voltage tracks the rod voltage over the full $14 \mathrm{mV}$ range of rod plateau responses, extending from an average rod membrane potential in darkness of -42 to $-56 \mathrm{mV}$. These absolute values are important in relation to the degree of activation of the L-type $\mathrm{Ca}$ current of the rod (see below).

\section{Rod voltage and glutamate release}

If synaptic transfer occurs over the full range of rod plateau voltages, glutamate release by rods would be expected to show a corresponding voltage dependence. We (Schmitz and Witkovsky, 1996, 1997) used a photoreceptor preparation (the reduced retina) developed by Cahill and Besharse (1991), which allows us to measure light-dependent glutamate release from photoreceptors. We used this data to relate glutamate release to changes in rod membrane voltage evoked by light and to the L-type Ca current in rods (Corey et al., 1984).

\section{Modulation by light}

Given that the Xenopus retina contains both rods and cones (Saxen, 1954) we used a spectral sensitivity test to evaluate whether the glutamate release by reduced retinas emanated from one or both photoreceptor classes. In the Xenopus retina the principal rod (Witkovsky et al., 1981) is 100 times more sensitive to $567 \mathrm{~nm}$ than to $660 \mathrm{~nm}$ light, whereas the principal, red-sensitive cone (Witkovsky et al., 1981) is about equally stimulated by these two wavelengths. A minority rod, which is most sensitive to blue light, constitutes $\sim 3 \%$ of the rod population (Denton and Pirenne, 1952) and has been ignored for the purposes of the present study. If glutamate is mainly released by cones, one expects identical release and quantal flux curves for the red and green lights, whereas if only rods release glutamate, the red curve should be displaced by $2 \log$ units to the right of the green curve on the intensity axis. If both photoreceptors contribute to release, the result should be somewhere in between these extremes.

For this test, reduced retinas, which showed a robust dark adaptation (dark efflux more than two times efflux in white light of $40 \mu \mathrm{W} / \mathrm{cm}^{2}$ ) were exposed to red or green background lights (five different quantal fluxes) for $20 \mathrm{~min}$ before two $10 \mathrm{~min}$ samples were taken. The preparations were dark-adapted between light exposures, and only data from preparations with a stable dark release were used ( $n=10$ eyes; $4-12$ samples for each intensity). Figure 5 illustrates the degree to which glutamate efflux (left vertical axis) was reduced by green (open squares) or red (closed squares) background lights. The data for red stimuli are displaced by $2 \log$ units to the right along the intensity axis with respect to the green light data, permitting the conclusion that all of the light-dependent glutamate efflux comes from rods.

For comparison, the mean plateau voltage to which the rod membrane is hyperpolarized by the same lights used to test glutamate release is plotted in Figure 5 (open triangles for the green, closed triangles for the red lights). These data were taken from Schmitz and Witkovsky (1996, their Figure 3) but here are given in absolute voltages, with the rod membrane potential in darkness set at $-42 \mathrm{mV}$. Because steady lights were used to evoke glutamate release, whereas rod light-evoked responses were obtained with $200 \mathrm{msec}$ flashes, we compared rod flash responses with those elicited by a light step (Fig. $5 B)$. The results $(n=15)$ indicate that the plateau voltage (arrowheads) estimated from brief flashes does correspond to the maintained plateau voltage elicited by a step of light.

Because both red and green lights appear to elicit glutamate release only from rods, the data from the two spectral stimuli were combined in Figure 6, in which mean relative glutamate release is plotted as a function of rod voltage. In a previous study (Schmitz and Witkovsky, 1996) we found that when the reduced retina is exposed to a saturating light, there is a baseline release of glutamate that is calcium independent. The increase in glutamate release over the baseline level, which occurs in darkness, however, depends on dihydropyridine-sensitive calcium channels (Schmitz and Witkovsky, 1997), in agreement with patch-clamp studies showing that amphibian rods possess an L-type Ca current (Bader et al., 1982; Corey et al., 1984). The continuous line in Figure 6 is the Boltzmann function for the L-type Ca current of rods, taken from Corey et al. (1984). The calcium current function was positioned vertically and scaled to give the best fit to the data points. The value of 1.0 on the scale of ordinates corresponds to the calcium-independent baseline glutamate release (Schmitz 


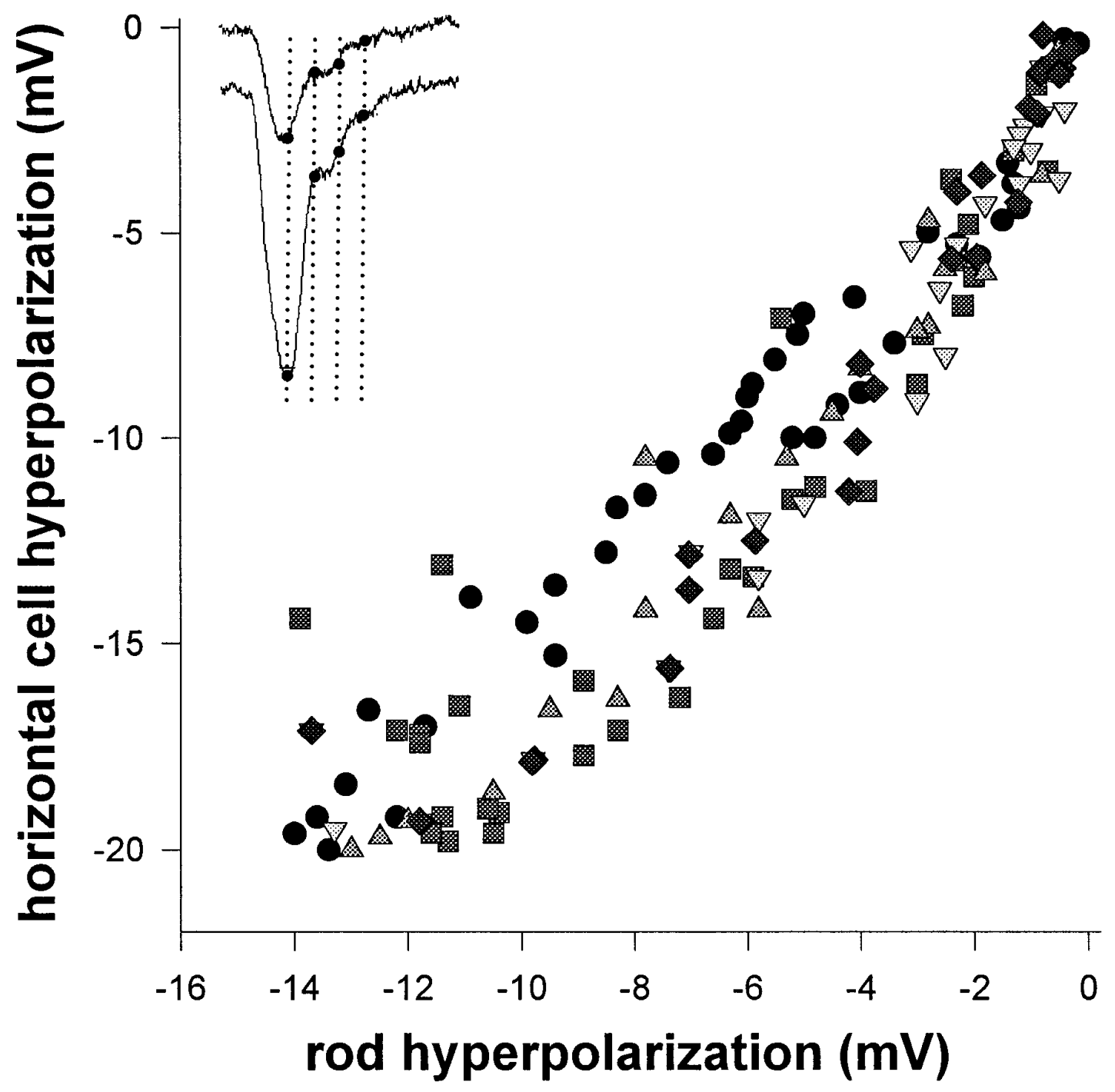

Figure 4. Temporal relation of rod and $\mathrm{HC}$ waveforms in scotopic state. The temporally corresponding voltages of five fully dark-adapted rod-HC pairs are plotted. Each symbol represents data points from a different rod-HC pair. For each rod-HC pair data were taken from responses to weak, intermediate, and bright test stimuli. Inset, Method: verticals are dropped through temporally aligned rod (top) and HC (bottom) light-evoked responses. The thicker dots indicate the voltage values used in the graph. Each vertically aligned pair of dots becomes one point in a two-dimensional matrix of rod versus $\mathrm{HC}$ voltage. Intervals between measures are $\sim 150 \mathrm{msec}$ in regions of rapid change and $\sim 500 \mathrm{msec}$ during maintained plateaus. Note that the points fall around a single function, the slope of which equals chord gain.

and Witkovsky, 1996). The reduction of glutamate release from 1.3 (corresponding to a rod voltage of $-56 \mathrm{mV}$ ) to 1.0 was evoked only by a bright white light $\left(40 \mu \mathrm{W} / \mathrm{cm}^{-2}\right)$ that did not further hyperpolarize the rod. Therefore this component of light-induced reduction of glutamate release might be attributable to cones. The shape of the calcium current function provides an excellent fit to the data for the range of rod plateau voltages over which the red and green test lights modulated glutamate release ( -42 to $-56 \mathrm{mV}$ ), indicating that steady-state glutamate release by rods is controlled by the L-type Ca current.

\section{A model for gain at the rod output synapse}

We now attempt to account for the steady-state relation between rod membrane potential and horizontal cell membrane potential. This relationship determines the steady-state gain of synaptic transmission between rods and HCs. The analysis combines results of our measurement of the dependence of glutamate release on rod membrane potential with known physiology of the glutamate conductance of the HC membrane. Our analysis is similar to that of Attwell (1990) but differs with respect to the dependence of transmitter release on calcium concentration. This is an important difference to which we return in Discussion. We will show that the dependence of glutamate release on rod membrane potential measured in the reduced preparation (Fig. 5) can account for the relationship between steady rod and $\mathrm{HC}$ voltages.

In our analysis, we will ignore any voltage-gated conductances on the assumption that they are relatively small over the range of $\mathrm{HC}$ voltages considered. Xenopus $\mathrm{HCs}$ have a glycine-gated $\mathrm{Cl}$ conductance (Stone and Witkovsky, 1984) and may also have a small GABA-gated Cl conductance (Witkovsky and Stone, 1987). In the absence of any detailed information on the dependence of the $\mathrm{HC} \mathrm{Cl}$ conductance on light level in this preparation, we begin 
Figure 5. Relation between rod voltage and glutamate release. $A$, Data from two separate experiments are combined. Triangles show the absolute rod plateau voltage (right scale of ordinates) induced by $567 \mathrm{~nm}$ or $660 \mathrm{~nm}$ stimuli, the log intensity of which is given on the scale of abscissa. Squares illustrate the fractional reduction in glutamate release (left scale of ordinates) induced by exposure to 567 or $660 \mathrm{~nm}$ lights of different log quantal flux indicated on the abscissa. Symbols are labeled on graph. $B$, Rod responses to flashes and steps of light. Rods were stimulated with a $200 \mathrm{msec}$ flash followed by a $5 \mathrm{sec}$ light step, both stimuli $555 \mathrm{~nm}$ light. The lowest trace is the stimulus marker. Log quantal flux for weakest stimulus (topmost trace) is 8.83 , increasing by $0.4 \log$ unit from top to bottom. For weaker stimuli, voltages of peak of flash and maintained plateau of step responses correspond. For brighter flashes, maintained step voltages correspond to inflection points (arrowheads) in flash responses.

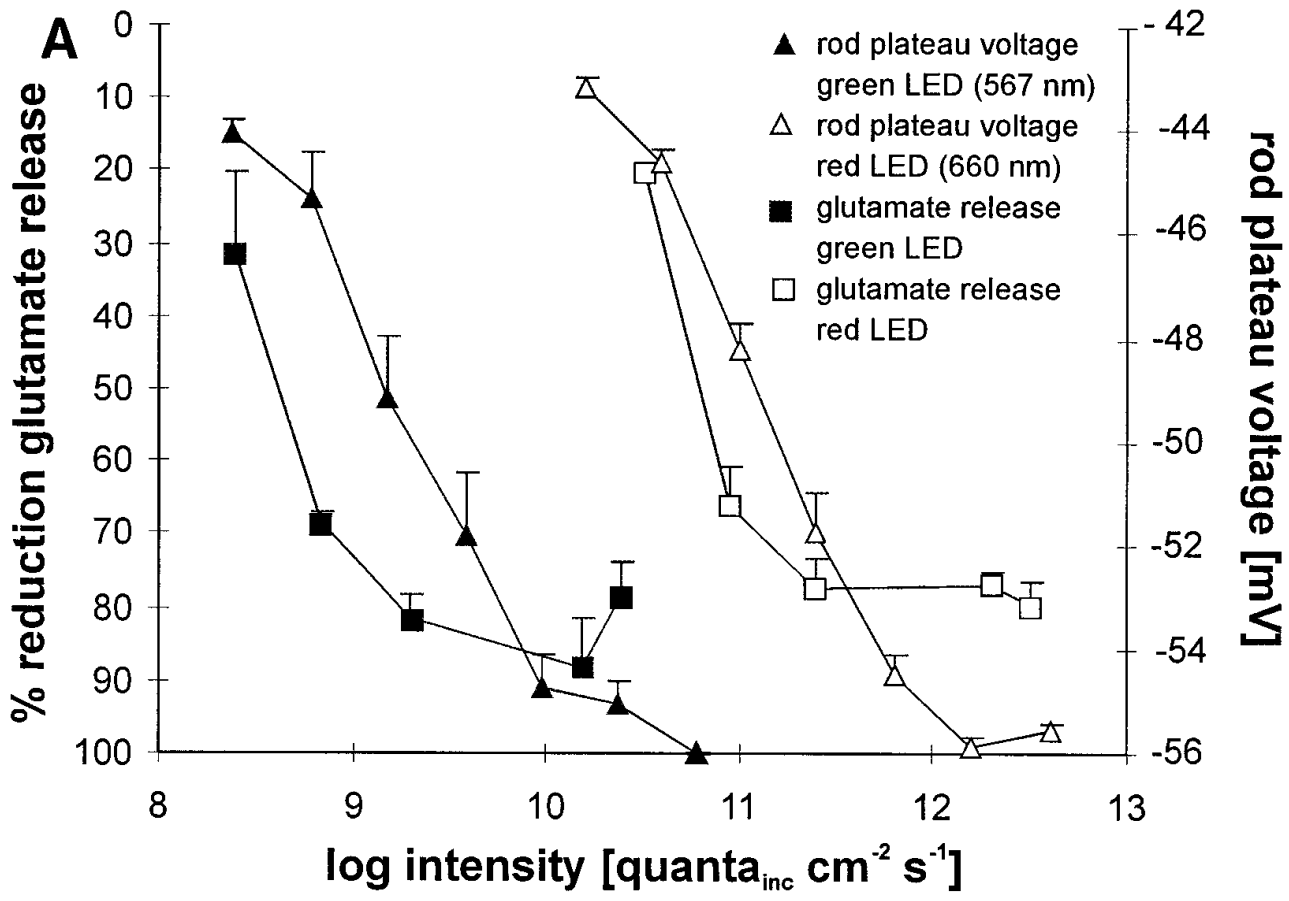

B

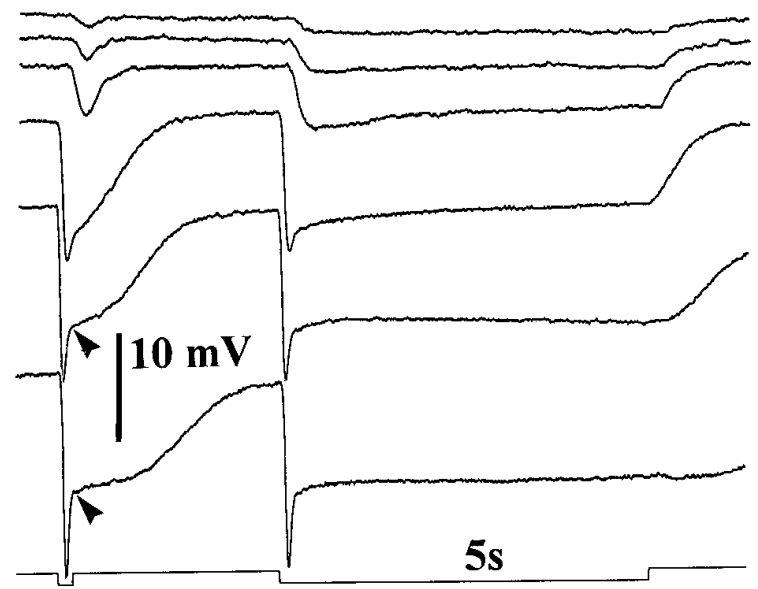

by making the simplifying assumption that the $\mathrm{Cl}$ conductance is constant. We also assume that the $\mathrm{K}$ conductance is constant.

In the steady state, the $\mathrm{HC}$ membrane potential, $u$, is given by:

$$
u=\left(G_{\mathrm{s}} E_{\mathrm{s}}+G_{\mathrm{r}} E_{\mathrm{r}}\right) /\left(G_{\mathrm{s}}+G_{\mathrm{r}}\right),
$$

where $G_{\mathrm{s}}$ is the glutamate-gated conductance; $E_{\mathrm{s}}$ is the reversal potential of corresponding postsynaptic current; $G_{\mathrm{r}}$ is the net conductance for $\mathrm{K}$ and $\mathrm{Cl}$; and $E_{\mathrm{r}}$ is the reversal potential for the net current through $\mathrm{Cl}$ and $\mathrm{K}$ channels. Then $G_{\mathrm{r}}=G_{\mathrm{K}}+G_{\mathrm{Cl}}$, where $G_{\mathrm{K}}$ and $G_{\mathrm{Cl}}$ are the $\mathrm{K}$ and $\mathrm{Cl}$ conductances, respectively, and:

$$
E_{\mathrm{r}}=\left(G_{\mathrm{K}} E_{\mathrm{K}}+G_{\mathrm{Cl}} E_{\mathrm{Cl}}\right) /\left(G_{\mathrm{K}}+G_{\mathrm{Cl}}\right) .
$$

We assume that the glutamate-gated conductance depends on glutamate concentration in the synaptic cleft [Glut] according to the Hill equation, with Hill coefficient $n$ :

$$
G_{\mathrm{s}}=G_{\mathrm{s}}^{\max }[\mathrm{Glut}]^{n} /\left(\mathrm{K}_{\mathrm{Glut}}^{n}+[\mathrm{Glut}]^{n}\right),
$$

where $G_{\mathrm{s}}^{\max }$ is the maximum glutamate conductance, and $\mathrm{K}_{\mathrm{Glut}}$ is the glutamate concentration that gives a half-maximal glutamate conductance. Experimentally determined values for $n$ vary between 1 and 2 (Shiells et al., 1986). In our computations, we set $n$ equal to 1.5 .

We assume that, under physiological conditions, the dependence of the rate of glutamate release by rod synaptic terminals on rod voltage is proportional to the release rate measured in the reduced preparation (Fig. 5). We further assume that the total rate of loss of glutamate in the synaptic cleft by diffusion and uptake is proportional to the steady glutamate concentration in the cleft. Under these conditions, [Glut] is proportional to the rate of release $r(v)$ :

$$
\text { [Glut }]=\alpha r(v),
$$

where $v$ is the steady rod membrane potential; $\alpha$ is a constant; and $r(v)$ is the rate of glutamate release given by:

$$
r(v)=C\{1+\exp [(A-V) / B]\}^{-1}+r_{\mathrm{o}} .
$$




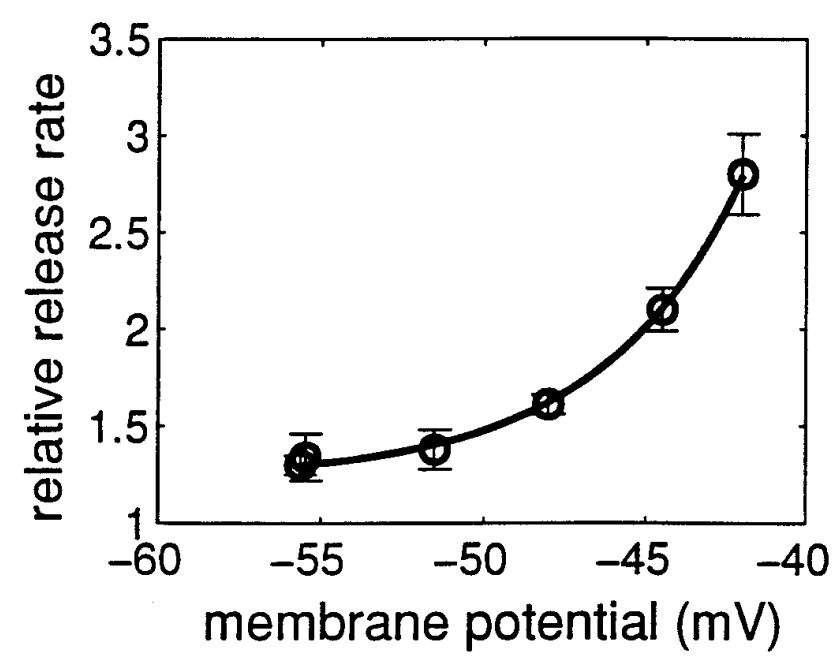

Figure 6. Relation of rod voltage and glutamate release to calcium current. The data points \pm SEM were obtained from the plots of Figure 5 by factoring out light intensity. The release versus voltage relation was nearly identical for 567 and $660 \mathrm{~nm}$ lights, so these data were combined and averaged. The points extend from the membrane potential of the rod in darkness $(-42 \mathrm{mV})$ to the maximum plateau value $(-56 \mathrm{mV})$ induced by a saturating light. The line through the points is the Boltzmann function for the L-type Ca current, $i / i_{\max }=[1+\exp (a-v / b)]^{-1}$, with the values for the half-saturation value, $a$, of $-22 \mathrm{mV}$ and the slope factor, $b$, of 4.3 taken from the data of Corey et al. (1984).

In Equation 5, $r_{\mathrm{o}}$ is the baseline rate of glutamate release; $C$ is a constant; $A$, the half-saturation value $(-22 \mathrm{mV})$, and $B$, the slope factor (4.3), are parameters in the Boltzmann activation function for the L-type Ca current. The specific values for $A$ and $B$ are taken from the study of Corey et al. (1984). Equation 5 was used to fit the glutamate release data in Figure 5.

It is important to note that the fact that the calcium-dependent transmitter release rate is proportional to the activation function for calcium current implies that transmitter release depends linearly on the calcium concentration in the terminal. This conclusion results from the fact that, if calcium is pumped out of the terminal at a rate proportional to the free concentration, then the free steady-state concentration will be proportional to the calcium current.

In the interests of computational efficiency, we express conductances and concentrations in dimensionless or normalized variables. If we define:

$$
z=[\text { Glut }] /[\text { Glut }]_{\text {dark }}
$$

then:

$$
z(v)=r(v) / r\left(v_{\text {dark }}\right)
$$

where [Glut $]_{\text {dark }}$ and $v_{\text {dark }}$ are the glutamate concentration and rod membrane potential in the dark. Let us also define:

$$
\begin{gathered}
k_{\mathrm{Glut}}=K_{\mathrm{Glut}} /[\mathrm{Glut}]_{\mathrm{dark}} ; \\
g_{\mathrm{s}}^{\max }=G_{\mathrm{s}}^{\max } / G_{\mathrm{r}} .
\end{gathered}
$$

Then the equation for the $\mathrm{HC}$ membrane potential can be written as:

$$
u=\left(g_{\mathrm{s}} E_{\mathrm{s}}+E_{\mathrm{r}}\right) /\left(g_{\mathrm{s}}+1\right),
$$

where $g_{\mathrm{s}}$ is the normalized glutamate-gated conductance; $g_{\mathrm{s}}=$ $G_{\mathrm{s}} / G_{\mathrm{r}}$. From Equation $10, g_{\mathrm{s}}$ can be written as:

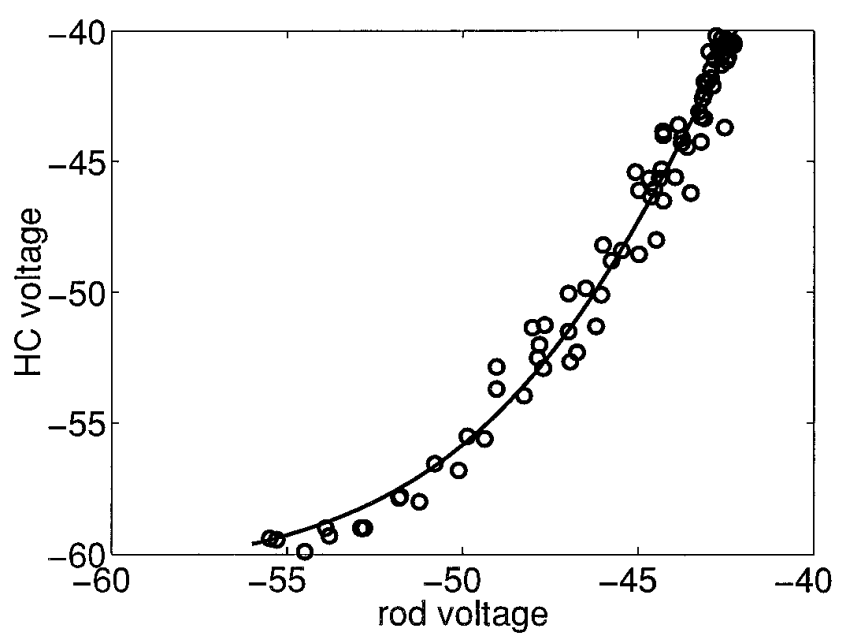

Figure 7. Model of rod versus horizontal cell light-induced potential changes. The data points are from one of the cells illustrated in Figure 4. Each cell in Figure 4 was similarly well fit by the continuous line, which is from a model described in Equation 10 of Results, which ties together the measurements of rod voltage, horizontal cell voltage, and glutamate release.

$$
g_{\mathrm{s}}=g_{\mathrm{s}}^{\max } z^{n}(v) /\left\{k_{\mathrm{Glut}}^{n}+z^{n}(v)\right\}
$$

Thus, Equations 10 and 11 express the HC voltage in terms of a known function of rod voltage, $z(v)$, and three unknown parameters, $g_{\mathrm{s}}^{\max }, \mathrm{k}_{\mathrm{Glut}}$, and $E_{\mathrm{r}}$. The reversal potential for the glutamategated current, $E_{\mathrm{s}}$, in Equation 10 above, is known to be close to zero (for review, see Wu, 1994).

We fit Equation 10 to our steady HC voltage versus rod voltage data, and the results are shown in Figure 7 . We set the Hill coefficient for the glutamate receptor $n$ equal to 1.5 and set $E_{\mathrm{r}}$ equal to $-86 \mathrm{mV}$, which is an estimate of $E_{\mathrm{K}}$ in this preparation. This value of $E_{\mathrm{r}}$ implies that $g_{\mathrm{C} 1}$ is small compared with $g_{\mathrm{K}}$. The parameters $g_{\mathrm{s}}$ and $k_{\mathrm{Glut}}$ were chosen to give the least sum of squared differences between the theoretical relation (Eq. 11) and the data. The best-fitting values of $g_{\mathrm{s}}$ and $k_{\mathrm{Glut}}$ were 3.1 and 1.4, respectively. A Hill coefficient of 2 worked about as well but required a more depolarized value of $E_{\mathrm{r}}$ of approximately -75 $\mathrm{mV}$. A Hill coefficient of 1 would fit the data only with an unphysiological value of $E_{\mathrm{r}}$ of approximately $-120 \mathrm{mV}$. The conclusion is that the steady-state rod-HC voltage relation can be accounted for reasonably well by the dependence of glutamate release on rod voltage (Fig. 6) that was measured in the reduced preparation.

One measure of the gain of synaptic transmission is the steadystate slope gain. The slope gain gives the increment in steady HC voltage per small increment in rod voltage. The steady-state slope gain is the derivative of the function that gives steady $\mathrm{HC}$ voltage as a function of steady rod voltage (Eq. 11). The steady-state slope gain can also be thought of as the value approached by the temporal transfer function linking rod voltage to $\mathrm{HC}$ voltage in the limit that the temporal frequency of modulation approaches zero. The steady-state slope gain derived from Equation 11 with the best-fitting parameters is plotted in Figure 8. This gain function has a maximum value of $\sim 2.8$ near the dark membrane potential of the rod, and the gain decreases smoothly to $\sim 0.3$ as the steady rod membrane potential approaches its maximum hyperpolarization of $\sim 14 \mathrm{mV}$. 


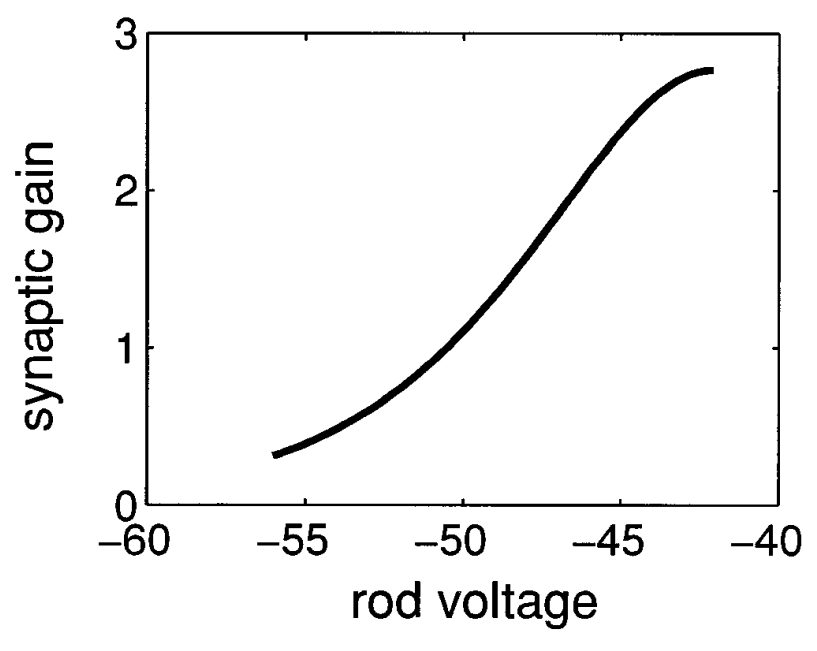

Figure 8. Gain of rod to HC synaptic transmission. Gain is computed as the derivative of the theoretical curve. This is the slope gain, generated from the model in the text, which gives, at each rod voltage operating point, the steady increment in $\mathrm{HC}$ voltage per small increment in rod voltage.

\section{DISCUSSION}

We derive two main conclusions from our data. First, in fully dark-adapted preparations, we showed that voltage modulates the glutamate release of the rod over its full range of light-evoked steady-state responses, extending from an average membrane potential in darkness of $-42 \mathrm{mV}$ to a maximum plateau of -56 $\mathrm{mV}$. The shape of the release versus voltage curve is fit by the activation curve of a high-voltage-activated, dihydropyridinesensitive, L-type Ca current, as defined by whole-cell patchclamp studies of rods in amphibian retinas (Corey et al., 1984; Wilkinson and Barnes, 1996). The correspondence between the activation function for the L-type $\mathrm{Ca}$ current and glutamate release indicates a linear relationship between them. If one assumes that $\mathrm{Ca}$ is pumped out of the terminal at a rate proportional to its concentration, the steady-state $\mathrm{Ca}$ concentration is proportional to the calcium current. This assumption leads to the conclusion that glutamate release by the rod terminal depends linearly on the $\mathrm{Ca}$ concentration.

The second point, which is in fact a corollary of the first, is that $\mathrm{HC}$ membrane potential is modulated over the full voltage range of rod function; i.e., there is a measurable increment of $\mathrm{HC}$ hyperpolarization for each increment of rod hyperpolarization evoked by light. The dimensionless steady-state slope gain of the rod to HC synapse is accounted for by the factors governing glutamate release by rods, if one assumes a Hill coefficient of 1.5 for the cooperativity of glutamate binding to its postsynaptic receptor. This cooperativity factor is consistent with a study of the dependence of $\mathrm{HC}$ voltage on exogenous glutamate in the dogfish retina (Shiells et al., 1986).

Previous studies of gain at the rod to HC synapse in amphibian retinas have been made by Attwell et al. (1987), Belgum and Copenhagen (1988), Wu (1988), and Yang and Wu (1996). All four reports agree that, for dim flash responses, the gain is relatively high, in the range of 4.5-9. The corresponding values for the Xenopus retina are lower (3-4), a difference that is explained, in part, by the smaller light-evoked $\mathrm{HC}$ responses we observed. This difference is related to the finding that, in darkness, Xenopus HCs are more hyperpolarized $(-40$ to $-45 \mathrm{mV})$ compared with the average value of -24.3 for toad (Belgum and Copenhagen, 1988) and either -32 (Attwell et al., 1987) or -18 $\mathrm{mV}$ (Wu, 1988) for salamander. Because bright light brings the $\mathrm{HC}$ membrane toward $E_{\mathrm{K}}$, a more hyperpolarized dark membrane potential restricts the range over which light can modulate $\mathrm{HC}$ voltage. Part of this difference in $\mathrm{HC}$ dark membrane potential may be attributable to the effect of $\mathrm{pH}$ on the L-type $\mathrm{Ca}$ current. Wu (1988) and Yang and Wu (1996) used a pH 7.7 buffer, compared with the $\mathrm{pH} 7.4$ Ringer's solution used in the present study. Barnes et al. (1993) have shown that high $\mathrm{pH}$ enhances the high-voltage-activated current in photoreceptors, thus leading to increased glutamate release and depolarization of the secondorder retinal neurons.

\section{Signal clipping at the rod synapse}

The cited studies and the present one are in agreement that rod to $\mathrm{HC}$ synaptic gain diminishes as stimulus intensity increases. Attwell et al. (1987) further observed that a strong rectification occurs at the rod synapse such that only rod voltages within $5 \mathrm{mV}$ of dark potential were effective in modulating glutamate release. They postulated that this synaptic rectification was a consequence of the activation function for the L-type Ca current controlling exocytosis. In fact, neither the data of Belgum and Copenhagen (1988) nor those of Wu (1988) concur in this finding; both studies show increments of $\mathrm{HC}$ voltage when rods are polarized beyond $5 \mathrm{mV}$ from their dark potential.

We have attempted to identify factors that might contribute to a reduced dynamic range of rod voltages that modulate the $\mathrm{HC}$ membrane voltage. The adaptational state of the retina appears to be the main contributory influence. Both Attwell et al. (1987) and Wu (1988) examined mesopic retinas in which both cone and rod inputs to the $\mathrm{HC}$ are apparent. We have reported (Witkovsky et al., 1989) that the cone to HC synapse diminishes the effectiveness of rod to $\mathrm{HC}$ communication, an effect explained, at least in part, by the shunting effect of the cone to HC synapse on rod to $\mathrm{HC}$ signal transfer. Under scotopic conditions, our data on rod-HC communication agree with those of Belgum and Copenhagen (1988). Their phase-plane plots of rod versus HC voltage show clearly that synaptic transmission continues for rod hyperpolarizations up to $-15 \mathrm{mV}$ from dark potential.

\section{The dependence of glutamate release on calcium}

There is general agreement that an L-type Ca current is intrinsic to photoreceptor inner segments (Bader et al., 1982; Corey et al., 1984; Barnes and Hille, 1989). This current is sensitive to dihydropyridines (Lasater and Witkovsky, 1991; Rieke and Schwartz, 1996; Wilkinson and Barnes, 1996). The effective operating range of the L-type Ca current is modified by pH (Barnes et al., 1993); accordingly we used the Boltzmann slope factor and halfactivation values from the study of Corey et al. (1984), because they were obtained at $\mathrm{pH} 7.3$, close to the $\mathrm{pH} 7.4$ bathing solution we used.

As discussed by Corey et al. (1984), in the salamander retinal rod the operating range of the L-type $\mathrm{Ca}$ current is far from the half-activation voltage of $-22 \mathrm{mV}$, resulting in a very small calcium current. Thus based on such recordings, it may appear that, at voltages hyperpolarized to $-45 \mathrm{mV}$, the calcium channels are effectively closed, as assumed by Attwell et al. (1987).

On the other hand, the small calcium influx is matched to the low total free calcium in the photoreceptor terminal (Rieke and Schwartz, 1996). There is good evidence that the L-type Ca current underlies glutamate release by rods. Rieke and Schwartz 
(1996) found that an increase in membrane capacitance, presumably reflecting net exocytosis (i.e., exocytosis - endocytosis) and $\mathrm{Ca}$ influx, increased in parallel in salamander rods, and that calcium entry was blocked by the dihydropyridine nisoldipine. Schmitz and Witkovsky (1997) reported that glutamate efflux from Xenopus photoreceptors is blocked by dihydropyridines but is not affected by blockers of $\mathrm{N}$ - or P-type calcium channels. The data for rods are similar to those for another nonspiking retinal cell, a depolarizing bipolar cell of the goldfish retina, for which it has been shown that exocytosis and glutamate release is gated by a dihydropyridine-sensitive L-type Ca current (Heidelberger and Matthews, 1992; Tachibana et al., 1993).

Another striking feature of the photoreceptor synapse is the apparent linear relation between calcium entry and capacitance change (Rieke and Schwartz, 1996). In our model (Equation 4) we assume a linear dependence of glutamate release on $\mathrm{Ca}$, consistent with the linear fit of the activation function for the L-type $\mathrm{Ca}$ current to the data relating glutamate release to rod voltage (Fig. 7). These data stand in contrast to those for spiking synapses, in which vesicle exocytosis occurs only for a very brief period related to the arrival of the spike (Llinas et al., 1995), and there is a high cooperativity (3-4) between $\mathrm{Ca}$ entry and transmitter release (Augustine et al., 1985).

\section{Comparison of models}

Our model for steady-state rod to $\mathrm{HC}$ synaptic transmission is qualitatively similar to that of Attwell (1990). The models, however, differ in details and give dramatically different rod-HC input-output functions. Our equation for calcium current in the synapse is the Boltzmann activation function used by Corey et al. (1984), rather than the exponential activation function of Bader et al. (1982) used by Attwell (1990). This is not an important difference, because the Boltzmann function is closely approximated by a simple exponential function over the physiological range of voltages. The major difference between the models lies in the dependence of transmitter release on the intracellular calcium concentration. To be consistent with our measurements of transmitter release (Schmitz and Witkovsky, 1996, 1997), we assume that the calcium-dependent release is simply proportional to the intracellular calcium concentration, and that there is a residual non-calcium-dependent release. The first of these assumptions is supported by the finding of Rieke and Schwartz (1996) of a parallel increase in intracellular calcium and membrane capacitance in salamander rods. Attwell (1990) assumed that transmitter release is proportional to the intracellular calcium concentration raised to the power $p$. One can infer from Attwell's stated values of other parameters that his value of $p$ is between 2 and 4.

In summary, our findings contribute to a growing body of data indicating that the photoreceptor synapse has special properties related to its tonic behavior. The rod output synapse is controlled by a sustained calcium current, which also has been shown to govern transmitter release at tonically active, retinal "on" bipolar cells (Heidelberger and Matthews, 1992; Tachibana et al., 1993).

\section{REFERENCES}

Attwell D (1990) The photoreceptor output synapse. Prog Ret Res 9:337-362.

Attwell D, Borges S, Wu SM, Wilson M (1987) Signal clipping by the rod output synapse. Nature 328:522-524.

Augustine GJ, Charlton MP, Smith SJ (1985) Calcium entry and trans- mitter release at voltage-clamped nerve terminals of squid. J Physiol (Lond) 367:163-181.

Bader CR, Bertrand D, Schwartz EA (1982) Voltage-activated and calcium-activated currents studied in solitary rod inner segments from the salamander retina. J Physiol (Lond) 331:253-284.

Barnes S, Hille B (1989) Ionic channels of the inner segment of tiger salamander cone photoreceptors. J Gen Physiol 94:719-743.

Barnes S, Merchant V, Mahmud F (1993) Modulation of transmission gain by protons at the photoreceptor output synapse. Proc Natl Acad Sci USA 90:10081-10085.

Belgum JH, Copenhagen DR (1988) Synaptic transfer of rod signals to horizontal and bipolar cells in the retina of the toad (Bufo marinus). J Physiol (Lond) 396:225-245.

Cahill GM, Besharse JC (1991) Resetting the clock in cultured Xenopus eyecups: regulation of retinal melatonin rhythms by light and D2 dopamine receptors. J Neurosci 11:2959-2971.

Cahill GM, Besharse JC (1992) Light-sensitive melatonin synthesis by Xenopus photoreceptors after destruction of the inner retina. Vis Neurosci 8:487-490.

Copenhagen DR, Jahr CE (1989) Release of endogenous excitatory amino acids from turtle photoreceptors. Nature 342:536-539.

Corey DP, Dubinsky JM, Schwartz EA (1984) The calcium current in inner segments of rods from the salamander (Ambystoma tigrinum) retina. J Physiol (Lond) 354:557-575.

Danbolt NC (1994) The high affinity uptake system for excitatory amino acids in the brain. Prog Neurobiol 44:377-396.

Denton EJ, Pirenne MN (1952) Green-coloured rods and retinal sensitivity. J Physiol (Lond) 116:33P.

Fosse VM, Kolstad J, Fonnum F (1986) A bioluminescence method for the measurement of L-glutamate: applications to the study of changes in the release of L-glutamate from lateral geniculate nucleus and superior colliculus after visual cortex ablation in rats. J Neurochem 47:340-349.

Hanani M, Vallerga S (1980) Rod and cone signals in the horizontal cells of the tiger salamander retina. J Physiol (Lond) 298:397-405.

Hare WA, Owen WG (1995) Similar effects of carbachol and dopamine on neurons in the distal retina of the tiger salamander. Vis Neurosci 12:443-455.

Heidelberger R, Matthews G (1992) Calcium influx and calcium current in single synaptic terminals of goldfish retinal bipolar cells. J Physiol (Lond) 447:235-256.

Katz E, Ferro PA, Cherksey BD, Sugimori M, Llinas R, Uchitel OD (1995) Effects of $\mathrm{Ca}^{2+} \mathrm{K}$ channel blockers on transmitter release and presynaptic currents at the frog neuromuscular junction. J Physiol (Lond) 486:695-706.

Krizaj D, Witkovsky P (1993) Effects of submicromolar concentrations of dopamine on photoreceptor to horizontal cell communication. Brain Res 627:122-128.

Krizaj D, Akopian A, Witkovsky P (1994) The effects of L-glutamate, AMPA, quisqualate, and kainate on retinal horizontal cells depend on adaptational state: implications for rod-cone interactions. J Neurosci 24:5661-5671.

Lasater EM, Witkovsky P (1991) The calcium current of turtle cone photoreceptor axon terminals. Neurosci Res [Suppl] 15:S165-S173.

Llinas R, Sugimori M, Silver RB (1995) The concept of calcium concentration microdomains in synaptic transmission. Neuropharmacology 34:1443-1451.

Marc RE, Liu W-LS, Kallionatis M, Raiguel SF, van Haesendonck E (1990) Patterns of glutamate immunoreactivity in the goldfish retina. J Neurosci 10:4006-4034.

Olivera BM, Miljanich GP, Ramachandran J, Adams ME (1994) Calcium channel diversity and neurotransmitter release: the $\omega$-conotoxins and $\omega$-agatoxins. Annu Rev Biochem 63:823-867.

Regehr WG, Mintz IM (1994) Participation of multiple calcium channel types in transmission at single climbing fiber to Purkinje cell synapses. Neuron 12:605-613.

Rieke F, Schwartz E (1994) A cGMP-gated current can control exocytosis at cone synapses. Neuron 13:863-873.

Rieke F, Schwartz E (1996) Asynchronous transmitter release: control of exocytosis and endocytosis at the salamander rod synapse. J Physiol (Lond) 493:1-8.

Saxen L (1954) The development of the visual cells. Embryological and physiological investigations on amphibia. Ann Acad Sci Fenn Ser A IV Biol 23:1-93.

Schmitz Y, Witkovsky P (1996) Glutamate release by the intact light- 
responsive photoreceptor layer of the Xenopus retina. J Neurosci Methods 68:55-60.

Schmitz Y, Witkovsky P (1997) Dependence of photoreceptor glutamate release on a dihydropyridine-sensitive calcium channel. Neuroscience 78:1209-1216.

Shiells RA, Falk G, Naghshineh S (1986) Ionotophoretic study of the action of excitatory amino acids on rod horizontal cells of the dogfish retina. Proc R Soc Lond [Biol] 227:121-135.

Stone S, Witkovsky P (1984) The actions of $\gamma$-aminobutyric acid, glycine and their antagonists upon horizontal cells of the Xenopus retina. J Physiol (Lond) 353:249-264.

Tachibana M, Takashi O, Arimura T, Kobayashi K, Piccolino M (1993) Dihydropyridine-sensitive calcium current mediates neurotransmitter release from bipolar cells of the goldfish retina. J Neurosci 13:2898-2909.

Wilkinson MF, Barnes S (1996) the dihydropyridine-sensitive calcium channel subtype in cone photoreceptors. J Gen Physiol 107:621-630.

Witkovsky P, Stone S (1987) GABA and glycine modify the balance of rod and cone inputs to horizontal cells in the Xenopus retina. J Exp Biol 47:13-22.

Witkovsky P, Levine JS, Engbretson GA, Hassin G, MacNichol Jr EF (1981) A microspectrophotometric study of normal and artificial visual pigments in the photoreceptors of Xenopus laevis. Vision Res $21: 867-873$

Witkovsky P, Stone S, Tranchina D (1989) Photoreceptor to horizontal cell synaptic transfer in the Xenopus retina: modulation by dopamine ligands and a circuit model for interactions of rod and cone inputs. J Neurophysiol 62:864-881.

Wu SM (1988) Synaptic transmission from rods to horizontal cells in dark-adapted tiger salamander retina. Vision Res. 28:1-8.

Wu SM (1994) Synaptic transmission in the outer retina. Annu Rev Physiol 56:141-165.

Yang X-L, Wu SM (1996) Response sensitivity and voltage gain of the rod- and cone-horizontal cell synapses in dark- and light-adapted tiger salamander retina. J Neurophysiol 76:3863-3874. 